\title{
Multisoliton perturbation theory for the Benjamin-Ono equation and its application to real physical systems
}

\author{
Y. Matsuno \\ Department of Physics, Faculty of Liberal Arts, Yamaguchi University, Yamaguchi 753, Japan
}

(Received 6 September 1994)

\begin{abstract}
A direct perturbation theory is developed to study the effects of small perturbations on the interaction process of algebraic solitons of the Benjamin-Ono (BO) equation. Using the method of multiple scales, the modulation equations for the amplitude and the phase of each soliton are derived in the lowest approximation. As practical applications of the theory, the interaction of two solitons is investigated for the two different types of perturbations that appear in real physical systems. One is a dissipative perturbation (BO-Burgers equation) and the other is a dispersive perturbation (higher-order BO equation). In both cases, the changes of the soliton parameters due to small perturbation are calculated by numerical integrations and their characteristics are elucidated in detail. Among them, the phase shift caused by the dispersive perturbation is a remarkable feature that has never been observed in the collision process of algebraic solitons.
\end{abstract}

PACS number(s): 03.40.Kf, 03.40.Gc, 02.90. $+\mathrm{p}, 02.60 .-\mathrm{x}$

\section{INTRODUCTION}

The development of the theory of nonlinear waves has enabled us to model real physical systems by simple nonlinear evolution equations (NEE's) called soliton equations [1-3]. The typical example is the Korteweg-de Vries $(\mathbf{K d V})$ equation that describes the unidirectional propagation of long waves of small amplitude. Almost all the NEE's thus derived incorporate the lowest-order nonlinearity in wave amplitude so that their applicability is severely restricted to small amplitude waves. In order to treat large amplitude waves, however, one must take into account the higher-order effects. In the context of water waves, various types of higher-order $\mathrm{KdV}$ equations have been derived in accordance with the physical situation under consideration, and their properties have been investigated in detail both analytically and numerically [4-11]. In the analytical approach, the higherorder terms are treated as perturbations and appropriate perturbation methods are applied. Several different approaches are known at present. These include a method based on the inverse-scattering transform (IST) [12-16], a direct method using multiple-time-scale expansion [17-20], a mixture of the above two methods [21-24], a technique using the variational principle [25], and a generalized reductive perturbation method $[5,6,26]$. These methods are reviewed critically in the literature [27,28] and hence their advantages are not discussed here. However, a brief review of the direct methods will be made in Sec. II D in connection with the present analysis.

In spite of a large number of works devoted to the study of the perturbation methods, there exist a few perturbed soliton equations that prevent us from applying the methods. A typical example is the following perturbed Benjamin-Ono (BO) equation:

$$
u_{t}+4 u u_{x}+H u_{x x}=\epsilon R[u], \quad u=u(x, t)
$$

Here $\epsilon R[u]$ represents the perturbation, $\epsilon$ is a small positive parameter that measures the magnitude of the perturbation, the operator $H$ is the Hilbert transform defined by

$$
H u(x, t)=\frac{1}{\pi} \mathrm{P} \int_{-\infty}^{\infty} \frac{u(y, t)}{y-x} d y,
$$

where the symbol $\mathbf{P}$ stands for the Cauchy principal value, and the subscripts $t$ and $x$ appended to $u$ denote partial differentiations. The BO equation has been derived by Benjamin [29] and later by Ono [30] to describe the propagation of long internal waves in a stratified fluid of great depth. The mathematical structure of the BO equation has been summarized in a textbook [31]. A novel feature when compared with the $\mathrm{KdV}$ equation is that the $\mathrm{BO}$ equation has a nonlocal dispersive term expressed by the Hilbert transform. Almost all the difficulties encountered in the analysis stem from this term.

The main purpose of this paper is to develop a direct perturbation theory of Eq. (1.1). In particular, we consider the effects of small perturbations on the interaction process of multiple solitons. In this respect, it should be remembered that most of the perturbation methods at hand deal only with the one-soliton problem in practice even though they may be applicable to the multisoliton problem as well [28]. In dealing with the latter problem analytically, great technical difficulties are usually accompanied. Our main concern here is the modification of the leading terms (solitons) due to the perturbation. Of course, it is important to estimate higher-order corrections, which are represented by the solutions of the inhomogeneous linear equations in the present perturbation scheme. But this problem will not be considered here.

This paper is organized as follows. In Sec. II, by developing a singular perturbation theory using a multipletime-scale expansion, we derive the system of modulation equations for the amplitude and the phase of each soliton. In Sec. III, the two practical applications are made for 
the different types of perturbations. One is concerned with the dissipative perturbation (BO-Burgers equation $[32,33])$ and the other deals with the dispersive perturbation (higher-order BO equation [11]). In both cases, the effects of small perturbations on the interaction process of the two solitons are investigated in detail by integrating numerically the NEE's that govern the time evolution of the soliton parameters. In Sec. IV, a brief summary of the main results obtained in the previous sections is made together with a future outlook. The three appendixes (A, $B$, and $C$ ) that follow will help the reader to understand the contents of the paper completely.

\section{DIRECT PERTURBATION THEORY}

\section{A. Method of multiple scales}

Following the standard procedure of the method of multiple scales $[34,35]$, we first introduce the different time scales $t_{j}$ by

$$
t_{j}=\epsilon^{j} t \quad(j=0,1,2, \ldots),
$$

and expand $u$ into an asymptotic series of the form

$$
u=\sum_{j=0}^{\infty} \epsilon^{j} u_{j}, \quad u_{j}=u_{j}\left(x, t_{0}, t_{1}, \ldots\right) .
$$

The time derivative is then transformed according to

$$
\frac{\partial}{\partial t}=\sum_{j=0}^{\infty} \epsilon^{j} \frac{\partial}{\partial t_{j}}
$$

Substituting (2.2) and (2.3) into (1.1) and equating coefficients of like powers of $\epsilon$, we obtain the system of equations for $u_{j}$, the first two members of which read as follows:

$$
\begin{aligned}
& u_{0, t_{0}}+4 u_{0} u_{0, x}+H u_{0, x x}=0, \\
& u_{1, t_{0}}+4\left(u_{0} u_{1}\right)_{x}+H u_{1, x x}=R\left[u_{0}\right]-u_{0, t_{1}} .
\end{aligned}
$$

Equation (2.4) is just the BO equation while $u_{j}$ with $j \geq 1$ satisfy the linear inhomogeneous equations.

\section{B. $N$-soliton solution}

In order to solve the above system of equations, we must first specify the solution of (2.4). We take for it the following $N$-soliton solution [36]:

$$
\begin{aligned}
& u_{0}=\frac{i}{2} \frac{\partial}{\partial x} \ln \left(f^{*} / f\right), \\
& f=\operatorname{det} M, \\
& M=\left(m_{j k}\right)=\delta_{j k}\left(i \theta_{j}+1\right)+2\left(1-\delta_{j k}\right) a_{j}\left(a_{j}-a_{k}\right)^{-1} \\
& \quad(j, k=1,2, \ldots, N), \\
& \theta_{j}=a_{j}\left(x-\xi_{j}\right), \quad \frac{\partial \xi_{j}}{\partial t_{0}}=a_{j} \quad(j=1,2, \ldots, N) .
\end{aligned}
$$

Here, $a_{j}$ and $\xi_{j}$ are the amplitude and the phase (or position) of the $j$ th soliton, respectively, $\delta_{j k}$ is Kronecker's delta, and the asterisk appended to $f$ denotes the complex conjugate. The amplitude parameters are all positive and satisfy the conditions $a_{j} \neq a_{k}$ for $j \neq k(j, k=1,2, \ldots, N)$. In the absence of the perturbation, $a_{j}$ is constant, independent of $t_{0}$ and $\xi_{j}=a_{j} t_{0}+\xi_{j 0}$ where $\xi_{j 0}$ is the initial phase of the $j$ th soliton. It is important to note that for large time, $u_{0}$ can be represented by a superposition of $N$ algebraic solitons as [36]

$$
u_{0} \sim \sum_{j=1}^{N} \frac{a_{j}}{a_{j}^{2}\left(x-\xi_{j}\right)^{2}+1} \quad\left(t_{0} \rightarrow \pm \infty\right) .
$$

The above expression shows that the $\mathrm{BO}$ solitons exhibit no phase shift after collision between them. This is a remarkable characteristic that has never been observed in the collision process of solitons expressed in terms of exponential functions. A detailed description of the interaction process of the BO solitons has been given in [37]. Now, due to the action of the small perturbation, the soliton parameters would be modulated slowly on the time scale of order $\epsilon^{-1}$. Hence, it is reasonable to assume that

$$
\begin{aligned}
& a_{j}=a_{j}\left(t_{1}, t_{2}, \ldots\right) \quad(j=1,2, \ldots, N), \\
& \xi_{j 0}=\xi_{j 0}\left(t_{1}, t_{2}, \ldots\right) \quad(j=1,2, \ldots, N) .
\end{aligned}
$$

Thus, the problem under consideration reduces to determining the time evolution of these parameters.

\section{Compatibility conditions}

With the $N$-soliton solution of Eq. (2.4), we are ready to solve Eq. (2.5). Though it is a linear equation, the nonlocal term expressed by the Hilbert transform makes the analytical treatment more difficult. Nevertheless, we can obtain the equations that determine the time evolution of the soliton parameters by requiring that the correction term $u_{1}$ is no more singular than the leading-order solution $u_{0}$. This condition turns out to be equivalent to the elimination of secular terms. To show this, let us first introduce the solutions $g_{j}$ of the adjoint equation for the homogeneous part of (2.5) by

$$
g_{j, t_{0}}+4 u_{0} g_{j, x}+H g_{j, x x}=0 \quad(j=1,2, \ldots) .
$$

Multiplying $g_{j}$ on both sides of (2.5) and integrating by parts with $x$, we obtain

$$
\begin{aligned}
\frac{\partial}{\partial t_{0}} \int_{-\infty}^{\infty} g_{j} u_{1} d x & =\int_{-\infty}^{\infty} g_{j}\left(R\left[u_{0}\right]-u_{0, t_{1}}\right) d x \\
& \equiv\left(g_{j}, R\left[u_{0}\right]-u_{0, t_{1}}\right)
\end{aligned}
$$

where use has been made of (2.9). As will be demonstrated below, $g_{j}$ can be represented by functionals of $u_{0}$. Hence, for sufficiently large time, $g_{j}$ depends on $x$ and $t_{0}$ through the combination $x-\xi_{j}$ [see (2.7)]. Then, the space integral in (2.10) can remove the $t_{0}$ dependence, resulting in the estimate $\int_{-\infty}^{\infty} g_{j} u_{1} d x \propto t_{0}\left(t_{0} \rightarrow \infty\right)$. This fact would imply the occurrence of secular terms in $u_{1}$. In order to eliminate such secular terms, we demand that the right-hand side of (2.10) vanish identically. To be more specific, these compatibility conditions become 


$$
\left(g_{j}, R\left[u_{0}\right]-u_{0, t_{1}}\right)=0 \quad(j=1,2, \ldots) .
$$

Since $u_{0}$ depends on $t_{1}$ through $a_{j}$ and $\xi_{j 0}$, one can write

$$
u_{0, t_{1}}=\sum_{s=1}^{N}\left(\frac{\partial a_{s}}{\partial t_{1}} \frac{\partial u_{0}}{\partial a_{s}}+\frac{\partial \xi_{s 0}}{\partial t_{1}} \frac{\partial u_{0}}{\partial \xi_{s 0}}\right) \text {. }
$$

Then, it readily follows from (2.11) and (2.12) that

$$
\sum_{s=1}^{N}\left[\left[g_{j}, \frac{\partial u_{0}}{\partial a_{s}}\right] \frac{\partial a_{s}}{\partial t_{1}}+\left[g_{j}, \frac{\partial u_{0}}{\partial \xi_{s 0}}\right] \frac{\partial \xi_{s 0}}{\partial t_{1}}\right]=\left(g_{j}, R\left[u_{0}\right]\right) \text {. }
$$

At this stage, we construct the solutions of (2.9) explicit1y. As in the case of the $\mathrm{KdV}$ equation [17], this can be achieved by taking

$$
\begin{aligned}
& g_{j}=\int_{-\infty}^{x} \frac{\partial u_{0}}{\partial a_{j}} d x \quad(j=1,2, \ldots, N), \\
& g_{j+N}=\int_{-\infty}^{x} \frac{\partial u_{0}}{\partial \xi_{j 0}} d x \quad(j=1,2, \ldots, N) .
\end{aligned}
$$

By virtue of (2.4), we can easily confirm that (2.14) indeed satisfy (2.9). In (2.14), $a_{j}$ and $\xi_{j 0}$ are taken as the independent parameters. In this situation, $g_{j}$ yields a term proportional to $t_{0} g_{j+N}$, since $u_{0, a_{j}}=\left.u_{0, a_{j}}\right|_{\theta_{j}}+\left(-\theta_{j} /\right.$ $\left.a_{j}^{2}+2 t_{0}\right) u_{0, \xi_{j 0}}$, where the first term on the right-hand side of this expression means the differentiation with $a_{j}$ while keeping $\theta_{j}$ constant. However, this term is not independent of (2.14b) and hence gives rise to no substantial contribution to the compatibility conditions. In order to avoid such an undesirable behavior of $g_{j}$, it is convenient to introduce the independent parameter $\xi_{j}$ instead of $\xi_{j 0}$ $(j=1,2, \ldots, N)$.

\section{Time evolution of the soliton parameters}

The final step in our perturbation scheme is to derive the time evolution equations of the soliton parameters. For this purpose, it is crucial to observe the following orthogonality relations, which can be obtained with the use of the BO equation and its explicit $N$-soliton solution (see Appendix A):

$$
\begin{aligned}
& {\left[g_{i}, \frac{\partial u_{0}}{\partial \xi_{j 0}}\right]=-\left[g_{i+N}, \frac{\partial u_{0}}{\partial a_{j}}\right]=\frac{\pi}{4} \delta_{i j}} \\
& (i, j=1,2, \ldots, N), \\
& {\left[g_{i}, \frac{\partial u_{0}}{\partial a_{j}}\right]=\left[g_{i+N}, \frac{\partial u_{0}}{\partial \xi_{j 0}}\right]=0 \quad(i, j=1,2, \ldots, N) .}
\end{aligned}
$$

Substituting (2.15) into (2.13), we arrive at the system of ordinary differential equations that govern the time evolution of $a_{j}$ and $\xi_{j 0}$. In the following, we consider $\xi_{j}$ instead of $\xi_{j 0}$. We write the resulting equations in terms of the original time variable by using (2.3) together with the relations $a_{j, t_{0}}=0, \xi_{j, t_{0}}=a_{j}(j=1,2, \ldots, N)$ as follows:

$$
\begin{aligned}
& \frac{d a_{j}}{d t}=-\frac{4 \epsilon}{\pi}\left(g_{j+N}, R\left[u_{0}\right]\right) \quad(j=1,2, \ldots, N), \\
& \frac{d \xi_{j}}{d t}=a_{j}+\frac{4 \epsilon}{\pi}\left(g_{j}, R\left[u_{0}\right]\right) \quad(j=1,2, \ldots, N) .
\end{aligned}
$$

The solutions of the above equations describe the slow changes of the amplitude and the phase of each soliton, which are induced by the perturbation. It should be emphasized that for the purpose of calculating the inner products in (2.16) and (2.17), we only need the information of the $N$-soliton solution, which can be usually obtained without recourse to IST. The leading-order solution $u_{0}$ is valid uniformly over the long-time interval of order $\epsilon^{-1}$. Beyond this interval, one must take into account the higher-order modulation effects. In other words, it is necessary to introduce other time scales $t_{2}, t_{3}, \ldots$, to keep the expansion uniformly valid.

The leading-order analysis developed here disregards the emission of radiation as well as the distortion of the shape of solitons due to perturbation. These effects can be elucidated by proceeding to a higher-order approximation, i.e., by solving Eq. (2.5) with $u_{0}$ being the $N$-soliton solution of Eq. (2.4). However, at present, we have no analytical means of resolving the problem. Concerning this point, the work of Keener and McLaughlin is worth remarking upon [21]. They developed a direct perturbation theory analogous to that presented here. They constructed Green's functions to solve the linearized equations with the aid of IST and calculated explicitly the first-order corrections to the soliton solutions of the nonlinear Schrödinger and sine-Gordon equations [21,38]. Recently, a similar direct approach has been introduced by Herman [22], Kalyakin [23], and Konotop and Vekslerchik [24] to study the higher-order effects. The essence of their method is to construct solutions of the linearized equations, which yield the correction terms by using the completeness theorems as well as the orthogonality relations for the eigenfunctions of the linear operators. It should be remarked that in the case of the perturbed $\mathrm{KdV}$ equation the corresponding linear operator is not self-adjoint, unlike the cases treated by Keener and McLaughlin. Nevertheless, the method works well and gives rise to the same result as that obtained by IST.

Finally, we comment on the work of Tanaka [17]. He developed a direct perturbation theory of the $\mathrm{KdV}$ equation and derived the compatibility conditions analogous to (2.13). However, he did not notice the orthogonality relations and hence could not simplify the modulation equations. This latter point is important, particularly in applications to concrete multisoliton problems.

\section{APPLICATIONS}

In this section, we shall apply the theory developed in Sec. II to the two different types of perturbations, i.e., the dissipative and dispersive perturbations. The perturbed soliton equations considered here stem from the real physical systems and hence have practical importance $[11,32,33]$. While the investigations of these equations have been done from both analytical and numerical points of view, they are mainly concerned with the one- 
soliton problem. Here, we shall study the effects of small perturbations on the interaction process of the two algebraic solitons. In particular, the net changes of the soliton parameters due to the interaction are investigated in detail by integrating numerically the time evolution equations (2.16) and (2.17).

\section{A. BO-Burgers equation}

As a typical example of a weakly dissipative perturbation, we consider the following $\mathrm{BO}-$ Burgers equation

$$
u_{t}+4 u u_{x}+H u_{x x}=\epsilon u_{x x} \text {. }
$$

The equation describes the propagation of long waves in a magnetic flux tube of the solar atmosphere [32]. It is also introduced as a model equation for the description of long internal waves in the stratified lower atmosphere when turbulent dissipation is significant [33].

\section{Two-soliton solution}

To begin with, we write the two-soliton solution of the $\mathrm{BO}$ equation in the form

$$
\begin{aligned}
u_{0} & =\frac{i}{2} \frac{\partial}{\partial x} \ln \left(f^{*} / f\right), \\
f & =-a_{1} a_{2}\left(x-x_{1}\right)\left(x-x_{2}\right) \\
& =-a_{1} a_{2}\left(x^{2}-s_{1} x+s_{2}\right),
\end{aligned}
$$

where $x_{1}$ and $x_{2}$ are complex functions of $t_{0}$, whose imaginary parts are positive, and $s_{1}$ and $s_{2}$ are elementa- ry symmetric polynomials of $x_{1}$ and $x_{2}$, given by

$$
\begin{aligned}
& s_{1}=x_{1}+x_{2}, \\
& s_{2}=x_{1} x_{2} .
\end{aligned}
$$

By comparing (2.6) and (3.2), one finds

$s_{1}=\xi_{1}+\xi_{2}+i \frac{a_{1}+a_{2}}{a_{1} a_{2}}$,

$s_{2}=\xi_{1} \xi_{2}-\frac{1}{a_{1} a_{2}}\left(\frac{a_{1}+a_{2}}{a_{1}-a_{2}}\right)^{2}+i \frac{a_{1} \xi_{1}+a_{2} \xi_{2}}{a_{1} a_{2}}$.

The functions $g_{j}$ and $g_{j+2}(j=1,2)$ are immediately constructed from (2.14) and (3.2) as

$$
\begin{aligned}
& g_{j}=\frac{i}{2} \frac{\partial}{\partial a_{j}} \ln \left(f^{*} / f\right) \quad(j=1,2), \\
& g_{j+2}=\frac{i}{2} \frac{\partial}{\partial \xi_{j}} \ln \left(f^{*} / f\right) \quad(j=1,2),
\end{aligned}
$$

where $\xi_{j}$ have been used as the phase parameters instead of $\xi_{j 0}$ [see a comment following (2.14)]. If we perform differentiations, we can obtain the explicit functional forms of $u_{0}, g_{j}$, and $g_{j+2}$. But these are not written here.

\section{Time evolution of the soliton parameters}

The space integrals in (2.16) and (2.17) can be carried out analytically with the use of the residue theorem. The detail of the calculations is given in Appendix B. We quote only the final results as follows:

$$
\begin{aligned}
& \frac{d a_{1}}{d t}=-\epsilon a_{1}^{3}-\frac{4 \epsilon a_{1}^{3} s(s-1)}{(s+1)^{4}\left(y^{2}+1\right)^{2}}\left[\left(s^{2}+s^{2}+s+3\right) y^{2}-s^{3}+5 s^{2}-s+3\right], \\
& \frac{d a_{2}}{d t}=-\epsilon a_{2}^{3}+\frac{4 \epsilon a_{2}^{3}(s-1)}{s(s+1)^{4}\left(y^{2}+1\right)^{2}}\left[\left(3 s^{3}+s^{2}+s+1\right) y^{2}+3 s^{3}-s^{2}+5 s-1\right], \\
& \frac{d \xi_{1}}{d t}=a_{1}-\frac{8 \epsilon a_{1} s y}{(s-1)(s+1)^{3}\left(y^{2}+1\right)^{2}}\left[(s+1)\left(s^{2}+s+1\right) y^{2}+s^{4}-4 s^{3}+9 s^{2}-s+1\right], \\
& \frac{d \xi_{2}}{d t}=a_{2}+\frac{8 \epsilon a_{2} y}{s(s-1)(s+1)^{3}\left(y^{2}+1\right)^{2}}\left[s(s+1)\left(s^{2}+s+1\right) y^{2}+s^{4}-s^{3}+9 s^{2}-4 s+1\right] .
\end{aligned}
$$

Here we have put

$$
\begin{aligned}
& s=a_{2} / a_{1}, \\
& y=a_{1} s(s-1)\left(\xi_{1}-\xi_{2}\right) /(s+1)^{2},
\end{aligned}
$$

for simplicity. In the absence of the interaction, i.e., in the one-soliton state, the time evolution equations for $a_{j}$ and $\xi_{j}$ follow readily from (3.5) as

$$
\begin{aligned}
& \frac{d a_{j}}{d t}=-\epsilon a_{j}^{3} \quad(j=1,2), \\
& \frac{d \xi_{j}}{d t}=a_{j} \quad(j=1,2),
\end{aligned}
$$

which are easily integrated to yield

$$
\begin{aligned}
& a_{j}(t)=\frac{a_{j}(0)}{\sqrt{1+2 \epsilon a_{j}^{2}(0) t}}(j=1,2), \\
& \xi_{j}(t)=\xi_{j}(0)+\frac{\sqrt{1+2 \epsilon a_{j}^{2}(0) t}-1}{\epsilon a_{j}(0)} \quad(j=1,2),
\end{aligned}
$$

where $a_{j}(0)$ and $\xi_{j}(0)\left(=\xi_{j 0}\right)$ are the initial values of the amplitude and the phase of the $j$ th soliton, respectively. Thus, the amplitude of each soliton is found to decrease gradually as time passes.

\section{Numerical analysis}

In order to study the effects of the small perturbation on the interaction process of the two solitons, we have integrated numerically the system of ordinary differential 
equations (3.5) by employing the Runge-Kutta-Gill method. Numerical calculations were performed for the following two cases:

case $(i): a_{1}(0)=0.6, \quad a_{2}(0)=1,2$,

$$
\xi_{1}(0)=0, \quad \xi_{2}(0)=-150,
$$

case (ii): $a_{1}(0)=0.3, a_{2}(0)=1.5$,

$$
\xi_{1}(0)=0, \quad \xi_{2}(0)=-300 .
$$

In both cases, the parameter $\epsilon$ was set to 0.001 . Case (i) simulates the interaction of solitons with small amplitude ratio while case (ii) corresponds to the interaction with large amplitude ratio. The phase parameters $\xi_{j}(0)$ are chosen such that the two solitons are sufficiently separated in their initial positions. We are concerned here with the net changes of the amplitude and the phase due to the interaction. For this purpose, it is appropriate to define the quantities

$$
\begin{aligned}
& \Delta a_{j}(t)=a_{j}(t)-a_{j}^{(0)}(t) \quad(j=1,2), \\
& \Delta \xi_{j}(t)=\xi_{j}(t)-\xi_{j}^{(0)}(t) \quad(j=1,2),
\end{aligned}
$$

where $a_{j}^{(0)}$ and $\xi_{j}^{(0)}$ represent the expressions of the righthand sides of $(3.8 \mathrm{a})$ and $(3.8 \mathrm{~b})$, respectively. Figure 1 shows the time evolution of $\Delta a_{j}(t)$ for case (i). It is seen that the amplitude of the larger soliton increases and that of the smaller soliton decreases after the overtaking collision. Figure 2 also represents the time evolution of $\Delta \xi_{j}(t)$ for case (i). We can observe that the larger soliton suffers a positive phase shift, while that of the smaller soliton is negative.

The figures corresponding to $\Delta a_{j}$ and $\Delta \xi_{j}$ for case (ii) are depicted in Figs. 3 and 4, respectively. The tendency of the changes of the soliton parameters is the same as that for case (i). In the two cases exemplified here, the collision of solitons would occur at $t \simeq 400$. The gradual changes of $\Delta a_{j}$ and $\Delta \xi_{j}$ are observed at $t=1000$, an upper time limit to which the present leading-order analysis can be applied. One reason for this phenomenon is that, because of the decrease of velocity of each soliton

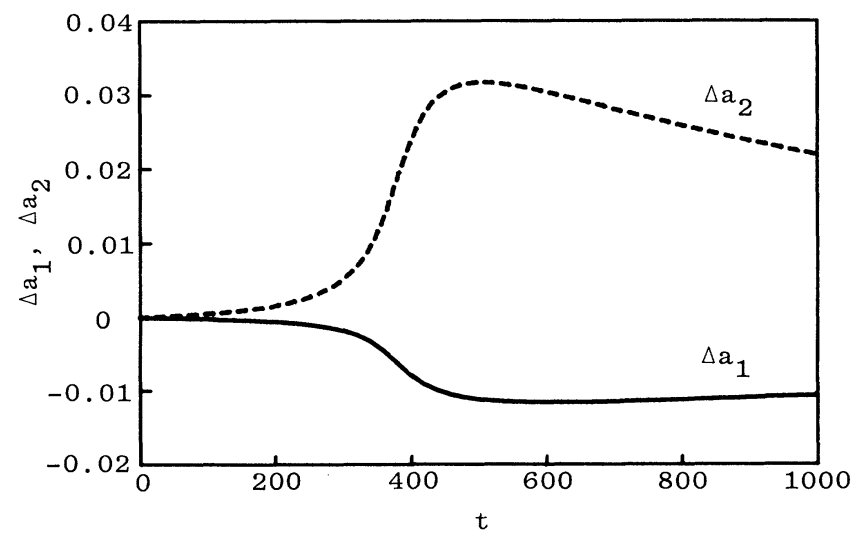

FIG. 1. Time evolution of $\Delta a_{1}$ and $\Delta a_{2}$ for case (i). The solid and broken lines represent $\Delta a_{1}$ and $\Delta a_{2}$, respectively.

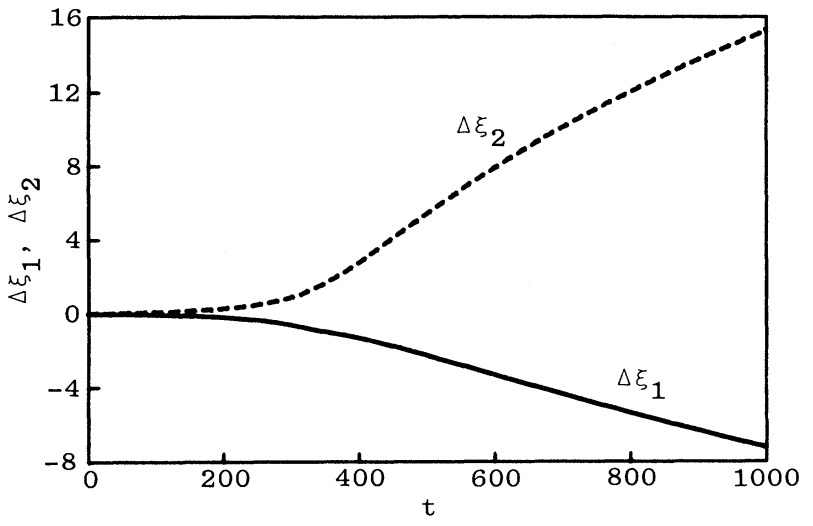

FIG. 2. Time evolution of $\Delta \xi_{1}$ and $\Delta \xi_{2}$ for case (i). The solid and broken lines represent $\Delta \xi_{1}$ and $\Delta \xi_{2}$, respectively.

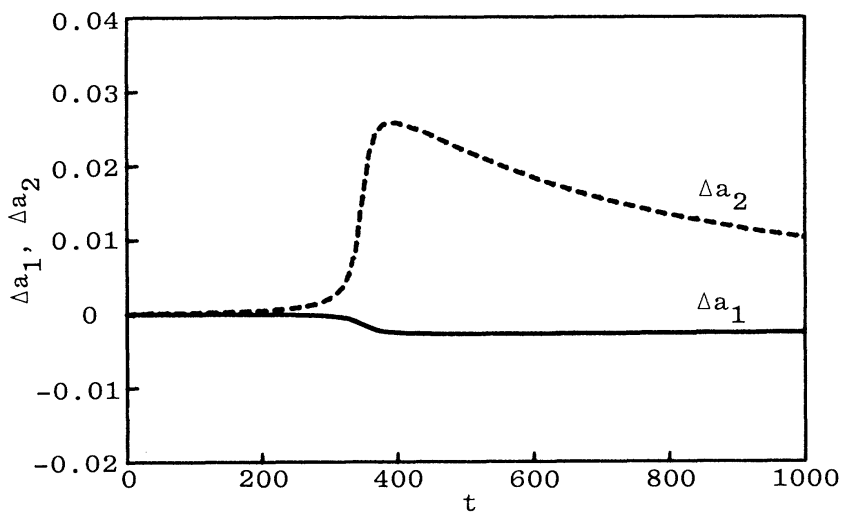

FIG. 3. Time evolution of $\Delta a_{1}$ and $\Delta a_{2}$ for case (ii).

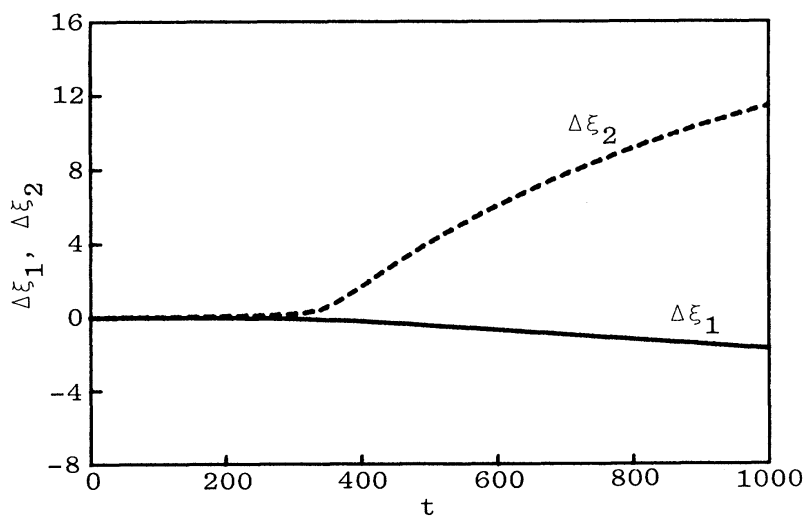

FIG. 4. Time evolution of $\Delta \xi_{1}$ and $\Delta \xi_{2}$ for case (ii). 
due to the dissipative perturbation, the duration of the interaction becomes longer when compared with that occurring in the absence of perturbation. Another reason may be attributed to the long-range tails of the BO solitons expressed by algebraic functions, since these prevent the solitons from separating perfectly from each other. We have also performed similar calculations for various amplitude ratio. However, the tendency mentioned above was not altered for all cases.

Although the present leading-order analysis clarifies the dominant behavior of the solution under small pertur- bation, it is important to estimate correction terms that stem from solutions of Eq. (2.5). In the case of the KdV equation with a small dissipation, it is well known that a shelf is formed in the lee of the solitary wave [13-15,39]. Whether a similar phenomenon occurs in the present situation is an important problem to be pursued in a future work.

\section{B. Higher-order BO equation}

The second application is made for the following higher-order BO equation:

$$
u_{t}+4 u u_{x}+H u_{x x}=\epsilon\left[3 u^{2} u_{x}-\frac{15}{4} u H u_{x x}-\frac{27}{4} H\left(u u_{x}\right)_{x}-3 u_{x} H u_{x}+\frac{27}{16 \Delta^{2}}\left[\Delta^{2}-\frac{4}{9}\right] u_{x x x}\right] .
$$

The equation has been derived to describe a unidirectional motion of interfacial waves in a two-layer fluid system in which the upper layer with a uniform density $\rho_{2}$ is infinitely deep and the depth of the lower layer with a uniform density $\rho_{1}$ is very small compared with the typical wavelength of the wave [11]. The parameter $\Delta$ is the density ratio $\rho_{2} / \rho_{1}$, which is assumed to be less than unity.

\section{Time evolution of the soliton parameters}

By evaluating the inner products in (2.16) and (2.17) with the formulas given in Appendix B, we obtain the following system of equations that govern the time evolution of the soliton parameters:

$$
\begin{aligned}
\frac{d a_{1}}{d t}= & \frac{3 \epsilon s^{2}(s-1)^{3} a_{1}^{4} y}{2 \Delta^{2}(s+1)^{5}\left(y^{2}+1\right)^{2}}\left[6\left(s^{2}+1\right)+\left(24 s^{2}+16 s+21\right) \Delta^{2}\right], \\
\frac{d a_{2}}{d t}= & \frac{-3 \epsilon(s-1)^{3} a_{2}^{4} y}{2 \Delta^{2} s^{2}(s+1)^{5}\left(y^{2}+1\right)^{2}}\left[6\left(s^{2}+1\right)+\left(21 s^{2}+16 s+24\right) \Delta^{2}\right] \\
\frac{d \xi_{1}}{d t}= & a_{1}-\frac{3 \epsilon}{16 \Delta^{2}}\left(6+31 \Delta^{2}\right) a_{1}^{2} \\
& -\frac{3 \epsilon s(s-1) a_{1}^{2}}{4 \Delta^{2}(s+1)^{4}\left(y^{2}+1\right)^{2}}\left[6\left\{(s-1)\left(s^{3}-s-2\right) y^{2}-s^{4}+5 s^{3}-3 s^{2}+5 s+2\right\}\right. \\
\frac{d \xi_{2}}{d t}= & a_{2}-\frac{3 \epsilon}{16 \Delta^{2}}\left(6+31 \Delta^{2}\right) a_{2}^{2} \\
& \left.\left.+\frac{3 \epsilon(s-1) a_{2}^{2}}{4 \Delta^{2} s^{2}(s+1)^{4}\left(y^{2}+1\right)^{2}}\left[6\left\{(s-1)\left(2 s^{3}+s^{2}-1\right) y^{2}+2 s^{4}+5 s^{3}-3 s^{2}+5 s-1\right\}\right)\left(24 s^{3}-35 s-44\right) y^{2}-24 s^{4}+88 s^{3}+7 s^{2}+129 s+44\right\} \Delta^{2}\right],
\end{aligned}
$$

In the absence of the interaction, these equations reduce to

$$
\begin{aligned}
& \frac{d a_{j}}{d t}=0 \quad(j=1,2) \\
& \frac{d \xi_{j}}{d t}=a_{j}-\frac{3 \epsilon}{16 \Delta^{2}}\left(6+31 \Delta^{2}\right) a_{j}^{2} \quad(j=1,2) .
\end{aligned}
$$

Integration of the above equations can be immediately done and the results are expressed as

$$
a_{j}(t)=a_{j}(0) \quad(j=1,2),
$$

$$
\begin{array}{r}
\xi_{j}(t)=\left[a_{j}(0)-\frac{3 \epsilon}{16 \Delta^{2}}\left(6+31 \Delta^{2}\right) a_{j}^{2}(0)\right] t+\xi_{j}(0) \\
(j=1,2)
\end{array}
$$

In contrast to the dissipative perturbation [see (3.8)], the amplitude does not change up to the approximation of $O(\epsilon)$, and the velocity of the soliton given by $d \xi_{j}(t) / d t$ has only a small correction. The net changes of the amplitude and the phase of each soliton are also defined by (3.9). In the present case, $a_{j}^{(0)}(t)$ and $\xi_{j}^{(0)}(t)$ are given by the expressions on the right-hand sides of (3.13a) and (3.13b), respectively. 


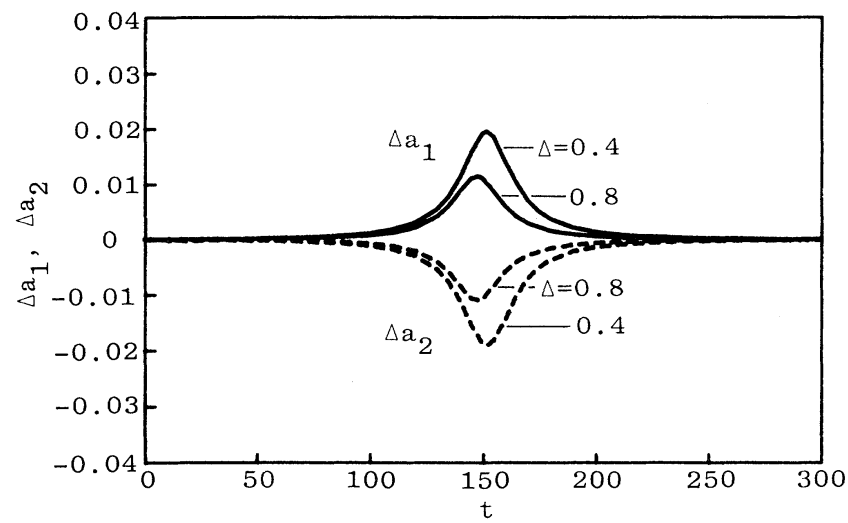

FIG. 5. Time evolution of $\Delta a_{1}$ and $\Delta a_{2}$ for case (i) and case (ii). The solid and broken lines represent $\Delta a_{1}$ and $\Delta a_{2}$, respectively.

\section{Numerical analysis}

Numerical calculations have been performed for the following four cases:

case (i): $\Delta=0.4, \quad a_{1}(0)=0.6, \quad a_{2}(0)=1.2$,

$$
\xi_{1}(0)=0, \quad \xi_{2}(0)=-85,
$$

case (ii): $\quad \Delta=0.8, \quad a_{1}(0)=0.6, \quad a_{2}(0)=1.2$,

$$
\xi_{1}(0)=0, \quad \xi_{2}(0)=-85,
$$

case (iii): $\Delta=0.4, a_{1}(0)=0.3, a_{2}(0)=1.5$,

$$
\xi_{1}(0)=0, \quad \xi_{2}(0)=-170,
$$

case (iv): $\Delta=0.8, a_{1}(0)=0.3, a_{2}(0)=1.5$,

$$
\xi_{1}(0)=0, \quad \xi_{2}(0)=-170 .
$$

In all cases, the parameter $\epsilon$ was set to 0.003 and the initial phases were chosen such that the collision of the two solitons occur at $t \simeq 150$. Figures 5 and 6 show the time evolution of $\Delta a_{j}(t)$ and $\Delta \xi_{j}(t)$, respectively, for case (i) and case (ii). The corresponding plots for case (iii) and case (iv) are also presented in Figs. 7 and 8, respectively.

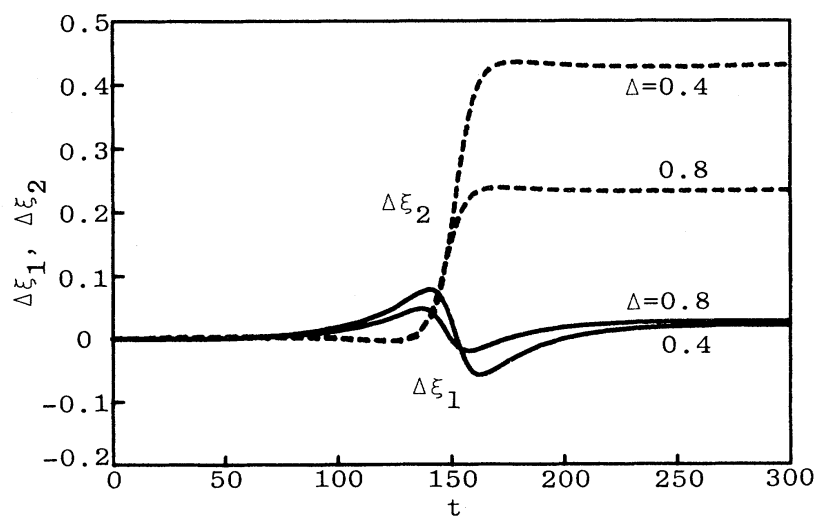
(ii).

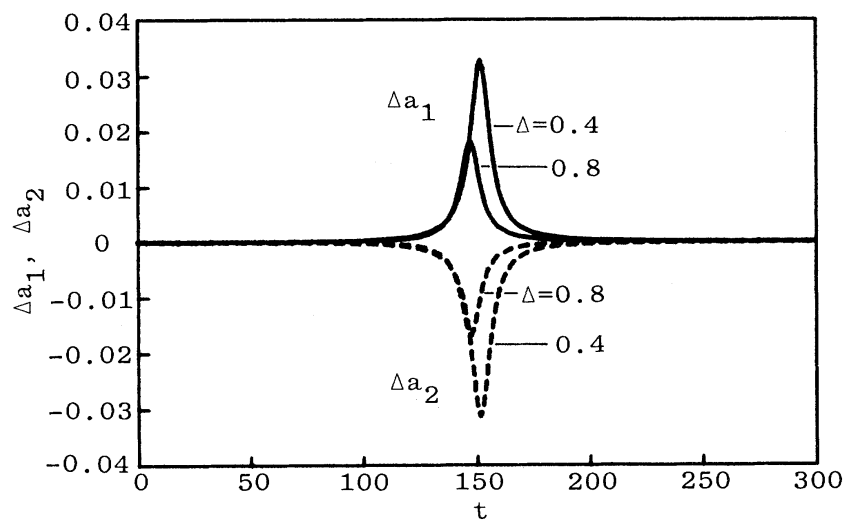

FIG. 7. Time evolution of $\Delta a_{1}$ and $\Delta a_{2}$ for case (iii) and case (iv).

From these figures, we see that the amplitudes of the solitons do not change after the interaction, but the phase shifts always occur, depending on both the initial values of the soliton parameters and the value of $\Delta$. Also we can observe that the maximum deviations of $\Delta a_{j}$ and $\Delta \xi_{j}$ decrease as the value of $\Delta$ increases. The time history of $\Delta a_{j}$ exhibits a similar profile for different amplitude ratio. However, a qualitative difference is found concerning the behavior of $\Delta \xi_{1}$ for the smaller soliton. Indeed, for a small amplitude ratio (see Fig. 6), as the solitons get close the smaller soliton is pushed forward before collision and then pulled backward after collision, thereby abruptly accelerating the larger soliton. As a result, the smaller soliton suffers a small but positive phase shift. For the large amplitude ratio, on the other hand, the reverse phenomenon occurs for the smaller soliton (see Fig. 8), i.e., the acceleration follows the deceleration and both effects lead to a negative phase shift.

\section{Detailed description of the phase shift}

In order to examine the feature of the phase shift in more detail, we shall solve (3.11) by means of the successive approximation and derive the formulas for the phase

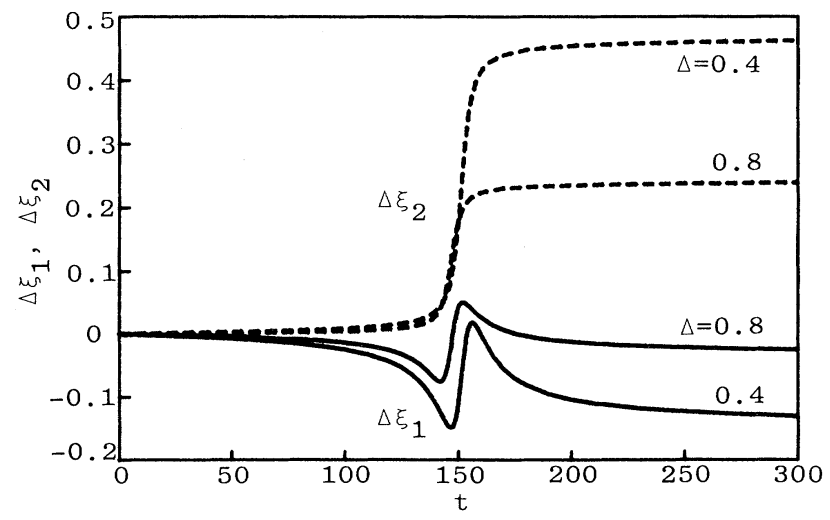
(iv). 
shifts, which are correct up to order $\epsilon$. First, we replace $a_{j}$ and $s$ in the $O(\epsilon)$ terms by their lowest-order approximations $a_{j}(0)$ and $s_{0}=a_{2}(0) / a_{1}(0)$, and then integrate with respect to $t$. The resulting expression for $a_{1}$ reads in the form

$$
a_{1}(t)=a_{1}(0)+\frac{3 \epsilon s_{0}\left(s_{0}-1\right) a_{1}^{2}(0)}{4 \Delta^{2}\left(s_{0}+1\right)^{3}}\left[6\left(s_{0}^{2}+1\right)+\left(24 s_{0}^{2}+16 s_{0}+21\right) \Delta^{2}\right]\left[\frac{1}{\tilde{y}^{2}+1}-\frac{1}{\tilde{y}_{0}^{2}+1}\right],
$$

where we have put

$$
\begin{aligned}
& \tilde{y}=a_{1}(0) s_{0}\left(s_{0}-1\right)\left[\left\{a_{1}(0)-a_{2}(0)\right\} t+\xi_{1}(0)-\xi_{2}(0)\right] /\left(s_{0}+1\right)^{2}, \\
& \tilde{y}_{0}=a_{1}(0) s_{0}\left(s_{0}-1\right)\left[\xi_{1}(0)-\xi_{2}(0)\right] /\left(s_{0}+1\right)^{2} .
\end{aligned}
$$

Integrating once again with $t$, we have

$$
\int_{0}^{t}\left[a_{1}(t)-a_{1}(0)\right] d t=\frac{3 \epsilon}{4 \Delta^{2}\left(s_{0}+1\right)\left(s_{0}-1\right)}\left[6\left(s_{0}^{2}+1\right)+\left(24 s_{0}^{2}+16 s_{0}+21\right) \Delta^{2}\right]\left[-\tan ^{-1} \tilde{y}+\tan ^{-1} \widetilde{y}_{0}+\frac{\tilde{y}-\widetilde{y}_{0}}{\widetilde{y}_{0}^{2}+1}\right] .
$$

Now, we can rewrite the phase shift defined by $(3.9 b)$ as

$$
\Delta \xi_{1}(t)=\int_{0}^{t}\left[\frac{d \xi_{1}}{d t}-\left[\frac{d \xi_{1}}{d t}\right] s_{1}\right] d t
$$

where $\left(d \xi_{1} / d t\right)_{s_{1}}$ means the soliton velocity in the absence of the interaction, and it is given explicitly by (3.12b) with $a_{1}=a_{1}(0)$. Substituting (3.11c) and (3.12b) into (3.16) and using (3.15), we arrive, after some calculations, at the following expression:

$$
\begin{aligned}
\Delta \xi_{1}(t)= & -\frac{3 \epsilon}{4 \Delta^{2}\left(s_{0}+1\right)^{2}}\left[6\left(s_{0}^{2}-2 s_{0}-1\right)+\left(8 s_{0}^{2}-46 s_{0}-23\right) \Delta^{2}\right]\left[\tan ^{-1} \widetilde{y}_{0}-\tan ^{-1} \widetilde{y}\right] \\
& +\frac{3 \epsilon}{4 \Delta^{2}\left(s_{0}+1\right)\left(s_{0}-1\right)}\left[6\left(s_{0}^{2}+1\right)+\left(24 s_{0}^{2}+16 s_{0}+1\right) \Delta^{2}\right] \frac{\widetilde{y}-\widetilde{y}_{0}}{\widetilde{y}_{0}^{2}+1} \\
& +\frac{3 \epsilon s_{0}}{4 \Delta^{2}\left(s_{0}+1\right)^{2}\left(s_{0}-1\right)}\left[6\left(s_{0}^{3}-3 s_{0}^{2}+s_{0}-3\right)+\left(24 s_{0}^{3}-56 s_{0}^{2}-21 s_{0}-69\right) \Delta^{2}\right]\left[\frac{\widetilde{y}_{0}}{\widetilde{y}_{0}^{2}+1}-\frac{\tilde{y}}{\widetilde{y}^{2}+1}\right] .
\end{aligned}
$$

The corresponding expression for $\Delta \xi_{2}(t)$ is obtained if we replace $s_{0}$ by $s_{0}^{-1}$ in (3.17). In Table $I$, the values of $\Delta \xi_{1}$ and $\Delta \xi_{2}$ evaluated by the above formula are compared with those by numerical calculations at $t=300$ for all cases. The quantitative agreement is fairly good except for a few values. If both solitons are separated sufficiently in their initial and final positions so that the second and third terms on the right-hand side of (3.17) can be neglected, (3.17) is considerably simplified as follows [40]:

$$
\begin{aligned}
\Delta \xi_{1}=-\frac{3 \pi \epsilon}{4 \Delta^{2}\left(s_{0}+1\right)^{2}} & {\left[6\left(s_{0}^{2}-2 s_{0}-1\right)\right.} \\
& \left.+\left(8 s_{0}^{2}-46 s_{0}-23\right) \Delta^{2}\right] .
\end{aligned}
$$

TABLE I. Comparison of the phase shift. The values in the first line of each entry are calculated by (3.17) and its counterpart for $\Delta \xi_{2}$, whereas the values in parentheses in the second line are obtained by numerical calculations.

\begin{tabular}{lcccc}
\hline \hline $\begin{array}{c}\text { Phase } \\
\text { shift }\end{array}$ & Case (i) & Case (ii) & Case (iii) & Case (iv) \\
\hline$\Delta \xi_{1}$ & 0.0182 & 0.0285 & -0.0846 & -0.0115 \\
& $(0.0193)$ & $(0.0267)$ & $(-0.131)$ & $(-0.0255)$ \\
$\Delta \xi_{2}$ & 0.395 & 0.214 & 0.411 & 0.220 \\
& $(0.430)$ & $(0.234)$ & $(0.462)$ & $(0.229)$ \\
\hline \hline
\end{tabular}

The corresponding expression of $\Delta \xi_{2}$ then takes the form

$$
\begin{aligned}
\Delta \xi_{2}=\frac{3 \pi \epsilon}{4 \Delta^{2}\left(s_{0}+1\right)^{2}} & {\left[6\left(s_{0}^{2}+2 s_{0}-1\right)\right.} \\
& \left.+\left(23 s_{0}^{2}+46 s_{0}-8\right) \Delta^{2}\right] .
\end{aligned}
$$

The phase shifts $\Delta \xi_{1}$ and $\Delta \xi_{2}$ are plotted in Fig. 9 as a function of $s_{0}$ for several values of $\Delta$, where the parameter $\epsilon$ has been taken to be 0.003 . One sees that the larger soliton always suffers a positive phase shift irrespective of the values of $s_{0}$ and $\Delta$. The situation is quite different for the smaller soliton. Indeed, $\Delta \xi_{1}$ changes sign according to the values of $s_{0}$ and $\Delta$. There exists a critical curve $\Delta$ vs $s_{0}$ that corresponds to $\Delta \xi_{1}=0$ (see Fig. 10). In the left region separated by the curve, $\Delta \xi_{1}>0$, whereas in the right region, $\Delta \xi_{1}<0$. However, for values of $s_{0}$ in the range $s_{0}>4.594, \Delta \xi_{1}$ always takes a negative value. The behavior of the phase shift described here is in agreement with the numerical results. Finally, it is worthwhile noting the prediction of the phase shift that takes place between algebraic solitons, since algebraic solitons found in various model NEE's never exhibit a phase shift after the interaction [41].

\section{SUMMARY AND OUTLOOK}

In this paper we have investigated the effects of small perturbations on the $N$-soliton solution of the BO equa- 


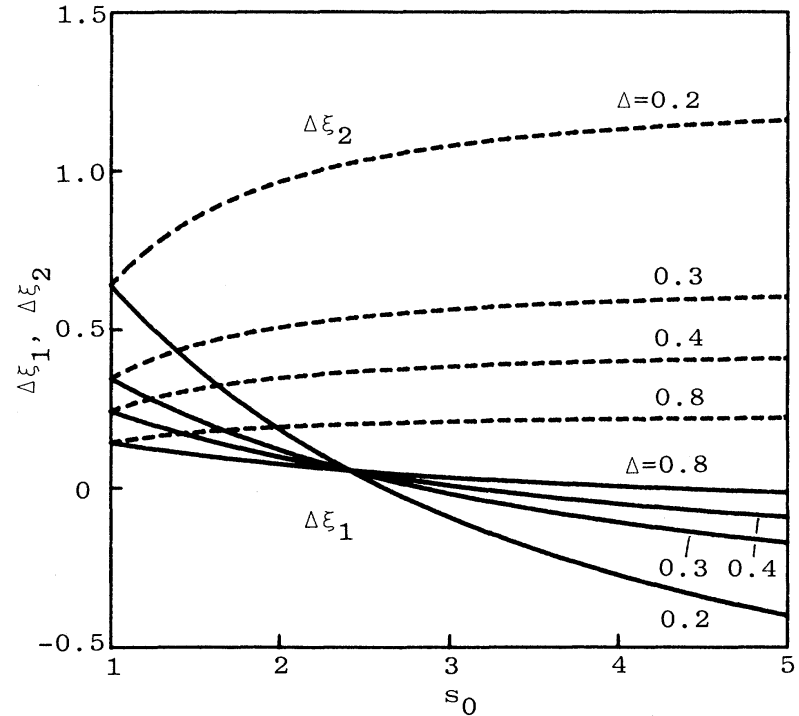

FIG. 9. Total phase shifts as a function of the initial amplitude ratio in the case of $\epsilon=0.003$ and $\Delta=0.2,0.3,0.4,0.8$. The solid and broken lines represent $\Delta \xi_{1}$ and $\Delta \xi_{2}$, respectively.

tion. A singular perturbation theory has been developed by employing the method of multiple scales. We have been concerned with the lowest-order approximation. Specifically, the modulation equations for the soliton parameters have been derived by imposing the compatibility conditions, which have been shown to be equivalent to the elimination of secular terms. Our approach is easy to follow, since it does not rely on IST as far as the leadingorder analysis is concerned. Practical applications were also done for the two different types of the perturbed $\mathrm{BO}$ equations, i.e., the BO-Burgers and higher-order BO equations. The former is a typical NEE with dissipative

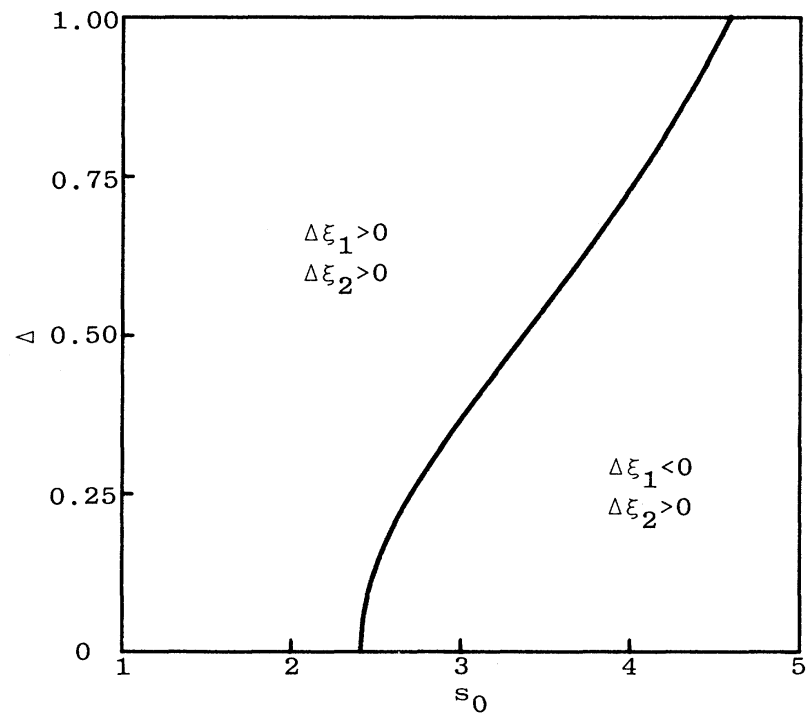

FIG. 10. Critical curve $\Delta$ vs $s_{0}$ that corresponds to $\Delta \xi_{1}=0$. perturbation while the latter is a typical NEE with dispersive perturbation. In both cases, the changes of the soliton parameters due to small perturbation were calculated by numerical integrations, and their characteristics were elucidated in detail. Among them is a remarkable feature, the phase shift caused by the dispersive perturbation, since it is the first example of what takes place between algebraic solutions. In any case, if we proceed to the next-order approximation, we must solve the linear inhomogeneous equation (2.5), with $u_{0}$ being the $N$ soliton solution. However, it seems to be quite a difficult problem within the framework of the present perturbation scheme. In this respect, it is worth mentioning that, in the case of the $\mathrm{KdV}$ equation, the linearized $\mathrm{KdV}$ equation, which is a counterpart of the homogeneous part of Eq. $(2.5)$, has been solved with the aid of IST $[42,43]$. The solution thus constructed has been used extensively to develop a direct perturbation theory for the $\mathrm{KdV}$ equation $[22,23]$. Whether a similar procedure is applicable to the linearized BO equation is an open but quite important question. Analytical predictions obtained in Sec. III may be confirmed by direct numerical simulations on the basis of Eqs. (3.1) and (3.10), as well as by an experiment analogous to that conducted for the purpose of determining the regions of applicability of various asymptotic theories dealing with finite-amplitude interfacial waves [44].

The method developed in this paper offers a powerful tool in analyzing perturbed NEE's, provided that they exhibit $\mathrm{N}$-soliton solutions in the absence of perturbations. For instance, one can apply the method to the following NEE:

$$
u_{t}+\alpha u u_{x}+\beta T u_{x x}=\epsilon R[u],
$$

where $T$ is an integro-differential operator with the property

$$
\int_{-\infty}^{\infty} f T g d x=-\int_{-\infty}^{\infty} g T f d x,
$$

for arbitrary functions $f$ and $g$ defined appropriately on the real axis, and $\alpha$ and $\beta$ are real constants. A special case of $T=H$ gives the perturbed BO equation. Another example is the perturbed $\mathrm{KdV}$ equation, which is obtained with $T=\partial / \partial x$. More generally, if we take

$$
\begin{aligned}
T u(x, t)=\frac{1}{2 \delta} \mathbf{P} \int_{-\infty}^{\infty} & {\left[\operatorname{coth}\left\{\frac{\pi}{2 \delta}(y-x)\right\}\right.} \\
& -\operatorname{sgn}(y-x)] u(y, t) d y,
\end{aligned}
$$

(4.1) becomes the finite-depth fluid equation (or the intermediate long wave equation) [45-48] with small perturbation. It reduces to the perturbed $\mathrm{KdV}$ equation in the shallow-water limit $\delta \rightarrow 0$ and to the perturbed BO equation in the deep-water limit $\delta \rightarrow \infty$. In all cases exemplified, the core of our approach is to construct explicitly solutions of linearized equations corresponding to (2.9) and to establish the orthogonality relations. In particular, the latter simplifies considerably the expressions of the modulation equations for the soliton parameters. 
In Appendix C, an application is made for the perturbed $\mathrm{KdV}$ equation and the modulation equations are derived explicitly for the $N$-soliton solution.

\section{APPENDIX A: ORTHOGONALITY RELATIONS}

The orthogonality relations [(2.15a) and (2.15b)] can be verified by using the $\mathrm{BO}$ equation and the asymptotic form of $N$-soliton solution (2.7). We begin with the proof of $(2.15 \mathrm{a})$. We first differentiate the inner product $\left(g_{i}, u_{0, \xi_{j 0}}\right)$ with $t_{0}$ and use (2.14a) to obtain

$$
\begin{aligned}
\frac{\partial}{\partial t_{0}}\left[g_{i}, \frac{\partial u_{0}}{\partial \xi_{j 0}}\right]=\int_{-\infty}^{\infty} & {\left[\frac{\partial u_{0}}{\partial \xi_{j 0}} \frac{\partial}{\partial a_{i}}\left(\frac{\partial \phi_{0}}{\partial t_{0}}\right)\right.} \\
& \left.+\frac{\partial \phi_{0}}{\partial a_{i}} \frac{\partial}{\partial \xi_{j 0}}\left(\frac{\partial u_{0}}{\partial t_{0}}\right)\right] d x,
\end{aligned}
$$

where the function $\phi_{0}$ has been defined by $u_{0}=\phi_{0, x}$, and it satisfies the equation

$$
\phi_{0, t_{0}}+2 u_{0}^{2}+H u_{0, x}=0 \text {. }
$$

Substituting (2.4) and (A2) into (A1) and integrating by parts, we find

$$
\frac{\partial}{\partial t_{0}}\left[g_{i}, \frac{\partial u_{0}}{\partial \xi_{j 0}}\right]=0,
$$

where use has been made of the relation $\int_{-\infty}^{\infty} f H g d x$ $=-\int_{-\infty}^{\infty} g H f d x$. (A3) implies that the inner product is independent of $t_{0}$. This fact allows us to evaluate it at $t_{0} \rightarrow \infty$, with the asymptotic form of $u_{0}$ given by (2.7) and that of $g_{i}$. The result is expressed as

$$
\begin{aligned}
& \left.\qquad g_{i}, \frac{\partial u_{0}}{\partial \xi_{j 0}}\right] \\
& \quad=\lim _{t_{0} \rightarrow \infty} \int_{-\infty}^{\infty} \frac{x-\xi_{i}-a_{i} t_{0}}{\left[a_{i}^{2}\left(x-\xi_{i}\right)^{2}+1\right]} \frac{2 a_{j}^{3}\left(x-\xi_{j}\right)}{\left[a_{j}^{2}\left(x-\xi_{j}\right)^{2}+1\right]^{2}} d x \\
& \quad=\pi\left(\frac{a_{j}}{a_{i}}\right]_{t_{0} \rightarrow \infty}^{2} \frac{-a_{j}^{2}\left(\xi_{i}-\xi_{j}\right)^{2}+\left[1+\frac{a_{j}}{a_{i}}\right]^{2}}{\left[a_{j}^{2}\left(\xi_{i}-\xi_{j}\right)^{2}+\left[1+\frac{a_{j}}{a_{i}}\right]^{2}\right]^{2}},
\end{aligned}
$$

where the integration has been performed using the residue theorem. If we note $\left|\xi_{i}-\xi_{j}\right| \rightarrow \infty$ as $t_{0} \rightarrow \infty$ for $i \neq j$, (A4) can be written compactly as

$$
\left[g_{i}, \frac{\partial u_{0}}{\partial \xi_{j 0}}\right]=\frac{\pi}{4} \delta_{i j} .
$$

It then turns out from (A5) and (2.14) that

$$
\left[g_{i+N}, \frac{\partial u_{0}}{\partial a_{j}}\right]=-\frac{\pi}{4} \delta_{i j},
$$

which, together with (A5), verifies (2.15a). By a similar argument, (2.15b) follows immediately.

\section{APPENDIX B: CALCULATION OF THE INNER PRODUCTS}

In deriving the modulation equations of the soliton parameters for the two types of perturbation investigated in Sec. II, it is necessary to calculate the inner products $\left(g_{i}, R\left[u_{0}\right]\right)$ and $\left(g_{i+N}, R\left[u_{0}\right]\right)$. Explicitly, they involve the integrals of the following forms:

$I_{n} \equiv \int_{-\infty}^{\infty} \frac{x^{n}}{\widetilde{f}(x)\left[\widetilde{f}^{*}(x)\right]^{3}} d x \quad(n=0,1, \ldots, 4)$,
$J_{n} \equiv \int_{-\infty}^{\infty} \frac{x^{n}}{\left[\widetilde{f}(x) \widetilde{f}^{*}(x)\right]^{2}} d x \quad(n=0,1, \ldots, 4)$,

where $\widetilde{f}(x)=\left(x-x_{1}\right)\left(x-x_{2}\right)$. These integrations can be carried out with the use of the residue theorem. For instance, if we recall $\operatorname{Im} x_{j}>0(j=1,2), I_{n}$ is evaluated by integrating it along a large semicircle in the upper half complex plane as

$$
\begin{aligned}
I_{n}=2 \pi i & {\left[\left.\frac{\partial}{\partial x} \frac{x^{n}}{\left(x-x_{2}\right)\left[\widetilde{f}^{*}(x)\right]^{3}}\right|_{x=x_{1}}\right.} \\
& \left.+\left.\frac{\partial}{\partial x} \frac{x^{n}}{\left(x-x_{1}\right)\left[\widetilde{f}^{*}(x)\right]^{3}}\right|_{x=x_{2}}\right] .
\end{aligned}
$$

The expression after performing the $x$ differentiation is found to be represented by $s_{1}\left(=x_{1}+x_{2}\right)$ and $s_{2}$ $\left(=x_{1} x_{2}\right)$ [see (3.2) and (3.3)]. The algebra involved is, however, quite cumbersome and hence it was managed with the aid of the algebraic programming system REDUCE. If we put $s_{1}=a+i b$ and $s_{2}=c+i d$, the final results are written as follows:

$$
\begin{gathered}
I_{0}=\frac{\pi}{4 h^{3}}\left[b\left\{-(a b)^{2}+3 a b d+b^{4}+b^{2} c-3 d^{2}\right\}\right. \\
\left.+i b\left(-2 a b^{3}+4 b^{2} d\right)\right] \\
I_{1}=\frac{\pi}{4 h^{3}}\left[-a b^{3} c+b^{4} d+3 b^{2} c d-d^{3}\right. \\
\left.\quad+i b^{2}\left(-a b d-b^{2} c+3 d^{2}\right)\right] \\
I_{2}=\frac{\pi}{4 h^{3}}\left[-a d^{3}-b^{3} c^{2}+b^{3} d^{2}+3 b c d^{2}\right. \\
\left.\quad+2 i b d\left(-b^{2} c+d^{2}\right)\right]
\end{gathered}
$$

$$
\begin{gathered}
I_{3}=\frac{\pi}{4 h^{3}}\left[d-(a d)^{2}+3 a b c d-3(b c)^{2}+(b d)^{2}+c d^{2}\right\} \\
\left.+i d^{2}\left(a b d-3 b^{2} c+d^{2}\right)\right]
\end{gathered}
$$

$$
\begin{aligned}
I_{4}=\frac{\pi}{4 h^{3}}[ & -(a d)^{3}+3 b c(a d)^{2}-3 a d(b c)^{2}+2 a c d^{3} \\
& \left.+(b c)^{3}-3 b(c d)^{2}+b d^{4}+2 i d^{3}(a d-2 b c)\right],
\end{aligned}
$$

$J_{0}=\frac{\pi}{2 h^{3}}\left[b\left(a^{2} b^{2}-3 a b d+b^{4}-b^{2} c+3 d^{2}\right)\right]$,

$J_{1}=\frac{\pi}{2 h^{3}}\left(a b^{3} c+b^{4} d-3 b^{2} c d+d^{3}\right)$, 


$$
\begin{aligned}
& J_{2}=\frac{\pi}{2 h^{3}}\left(a d^{3}+b^{3} c^{2}+b^{3} d^{2}-3 b c d^{2}\right) \\
& J_{3}=\frac{\pi}{2 h^{3}}\left[d\left\{(a d)^{2}-3 a b c d+3(b c)^{2}+(b d)^{2}-c d^{2}\right\}\right] \\
& J_{4}=\frac{\pi}{2 h^{3}}\left[a^{4} d^{3}-3 a^{3} b c d^{2}+3(a b c)^{2} d-3 a^{2} c d^{3}\right. \\
& \left.\quad-a(b c)^{3}+6 a b(c d)^{2}-3 b^{2} c^{3} d+c^{2} d^{3}+d^{5}\right] .
\end{aligned}
$$

Here in these expressions, $h$ is given by

$$
h=a b d-b^{2} c-d^{2} .
$$

Using these formulas, the inner products can be expressed as

$$
\begin{array}{r}
\left(g_{i}, R\left[u_{0}\right]\right)=\sum_{n=0}^{4}\left[\operatorname{Re}\left(\alpha_{n}^{(i)} I_{n}\right)+\left(\beta_{n}^{(i)} J_{n}\right)\right] \\
(i=1,2), \\
\left(g_{i+2}, R\left[u_{0}\right]\right)=\sum_{n=0}^{4}\left[\operatorname{Re}\left(\gamma_{n}^{(i)} I_{n}\right)+\left(\delta_{n}^{(i)} J_{n}\right)\right] \\
(i=1,2),
\end{array}
$$

where $\alpha_{n}^{(i)}, \beta_{n}^{(i)}, \gamma_{n}^{(i)}$, and $\delta_{n}^{(i)}$ are functions of the soliton parameters $a_{j}$ and $\xi_{j}(j=1,2)$, the explicit forms of which are, however, too complicated to write here. All the calculations in (B15) and (B16) have also been dealt with using REDUCE to obtain (3.5) and and (3.11).

\section{APPENDIX C: PERTURBED KdV EQUATION}

We write the perturbed $\mathrm{KdV}$ equation in the form

$$
u_{t}-6 u u_{x}+u_{x x x}=\epsilon R[u], \quad u=u(x, t) .
$$

The orthogonality relations corresponding to (2.15) are now written as

$$
\begin{aligned}
& {\left[g_{i}, \frac{\partial u_{0}}{\partial k_{j}}\right]=8 \delta_{i j} \quad(i, j=1,2, \ldots, N),} \\
& {\left[g_{i}, \frac{\partial u_{0}}{\partial \xi_{j 0}}\right]=-\left\lfloor g_{i+N}, \frac{\partial u_{0}}{\partial k_{j}}\right]=8 k_{i}^{2} \delta_{i j}} \\
& {\left[g_{i+N}, \frac{\partial u_{0}}{\partial \xi_{j 0}}\right]=0 \quad(i, j=1,2, \ldots, N),}
\end{aligned}
$$

where $k_{i}=k_{i}\left(t_{1}, t_{2}, \ldots\right)$ and $\xi_{i 0}=\xi_{i 0}\left(t_{1}, t_{2}, \ldots\right)$ are the soliton parameters and $u_{0}$ is the $N$-soliton solution of the $\mathrm{KdV}$ equation given explicitly by [49]

$$
\begin{aligned}
& u_{0}=-2(\ln f)_{x x}, \\
& f=\operatorname{det} M, \\
& M=\left(m_{i j}\right)=\delta_{i j}+\frac{2 \sqrt{k_{i} k_{j}} e^{-\left(\theta_{i}+\theta_{j}\right)}}{k_{i}+k_{j}} \\
& \quad(i, j=1,2, \ldots, N),
\end{aligned}
$$

$\theta_{i}=k_{i}\left(x-\xi_{i}\right), \quad \frac{\partial \xi_{i}}{\partial t_{0}}=4 k_{i}^{2} \quad(i=1,2, \ldots, N)$

In the following analysis, we introduce $k_{i}$ and $\xi_{i}$ as the independent soliton parameters instead of $k_{i}$ and $\xi_{i 0}$. If we use the compatibility conditions $(2.11)$ and the above orthogonality relations, we find that the soliton parameters evolve according to the following equations:

$$
\begin{array}{r}
\frac{d k_{i}}{d t}=-\frac{\epsilon}{8 k_{i}^{2}}\left(g_{i+N}, R\left[u_{0}\right]\right) \\
(i=1,2, \ldots, N), \\
\frac{d \xi_{i}}{d t}=4 k_{i}^{2}+\frac{\epsilon}{8 k_{i}^{2}}\left[g_{i}+\frac{g_{i+N}}{k_{i}^{2}}, R\left[u_{0}\right]\right) \\
(i=1,2, \ldots, N) .
\end{array}
$$

To calculate $g_{i}$ and $g_{i+N}$, we employ another useful expression of $u_{0}$ [49],

$$
u_{0}=-4 \sum_{i=1}^{N} k_{i} \phi_{i}^{2},
$$

where $\phi_{i}$ is the solution of the following system of linear algebraic equations:

$$
\begin{aligned}
\phi_{i}+\sum_{j=1}^{N} \frac{2 \sqrt{k_{i} k_{j}} e^{-\left(\theta_{i}+\theta_{j}\right)}}{k_{i}+k_{j}} \phi_{j} & =\sqrt{2 k_{i}} e^{-\theta_{i}} \\
(i & =1,2, \ldots, N) .
\end{aligned}
$$

The $\phi_{i}$ may be represented in terms of $f$ as

$$
\phi_{i}=\frac{1}{f} \sum_{j=1}^{N} \sqrt{2 k_{j}} e^{-\theta_{j}} \frac{\partial f}{\partial m_{j i}} .
$$

It now follows from the definition of $g_{i+N}$ [see (2.14)], (C5), and (C13) that

$$
\begin{aligned}
g_{i+N} & =-2(\ln f)_{x \xi_{i}} \\
& =2 k_{i} \sqrt{2 k_{i}} e^{-\theta_{i}} \phi_{i}+2 \sum_{j=1}^{N} \sqrt{2 k_{j}} e^{-\theta_{j}} \frac{\partial \phi_{j}}{\partial \xi_{i}} .
\end{aligned}
$$

Differentiating $(\mathrm{C} 12)$ with respect to $\xi_{i}$, we obtain

$$
\frac{\partial \phi_{j}}{\partial \xi_{i}}+\sum_{s=1}^{N} \frac{2 \sqrt{k_{j} k_{s}} e^{-\left(\theta_{j}+\theta_{s}\right)}}{k_{j}+k_{s}} \frac{\partial \phi_{s}}{\partial \xi_{i}}=G_{i j},
$$

where

$$
\begin{aligned}
G_{i j} \equiv & -\sum_{s=1}^{N} \frac{2 \sqrt{k_{j} k_{s}}}{k_{j}+k_{s}}\left(k_{j} \delta_{i j}+k_{s} \delta_{i s}\right) e^{-\left(\theta_{j}+\theta_{s}\right)} \phi_{s} \\
& +k_{j} \sqrt{2 k_{j}} e^{-\theta_{j}} \delta_{i j} .
\end{aligned}
$$

The solution $\partial \phi_{j} / \partial \xi_{i}$ of $(\mathrm{C} 15)$ can be expressed in terms of $m_{i j}$ and $G_{i j}$ by using Crammer's rule. If we insert the result into (C14), we obtain, after some algebra, the simple formula

$$
g_{i+N}=4 k_{i} \phi_{i}^{2} \quad(i=1,2, \ldots, N) .
$$

A similar calculation leads to the expression of $g_{i}$ as 


$$
g_{i}=-2(\ln f)_{x k_{i}}-4=-\frac{4}{k_{i}} \theta_{i} \phi_{i}^{2}+\frac{2}{k_{i}} \phi_{i}^{2}+8 \phi_{i} \sum_{j=1}^{N} \frac{\sqrt{k_{i} k_{j}}}{\left(k_{i}+k_{j}\right)^{2}} e^{-\left(\theta_{i}+\theta_{j}\right)} \phi_{j}-4 \quad(i=1,2, \ldots, N) .
$$

The time evolution of the soliton parameters is now found from (C9), (C10), (C17), and (C18) as follows:

$$
\begin{aligned}
\frac{d k_{i}}{d t} & =-\frac{\epsilon}{2 k_{i}} \int_{-\infty}^{\infty} \phi_{i}^{2} R\left[u_{0}\right] d x \quad(i=1,2, \ldots, N), \\
\frac{d \xi_{i}}{d t} & =4 k_{i}^{2}-\frac{\epsilon}{4 k_{i}^{3}} \int_{-\infty}^{\infty} R\left[u_{0}\right]\left[2 \theta_{i} \phi_{i}^{2}-3 \phi_{i}^{2}+2 k_{i}-4 \sum_{j=1}^{N} \frac{k_{i} \sqrt{k_{i} k_{j}}}{\left(k_{i}+k_{j}\right)^{2}} e^{-\left(\theta_{i}+\theta_{j}\right)} \phi_{i} \phi_{j}\right] d x \quad(i=1,2, \ldots, N) .
\end{aligned}
$$

In the simplest one-soliton case, $u_{0}$ and $\phi_{1}$ are given by

$$
\begin{aligned}
& u_{0}=-2 k_{1}^{2} \operatorname{sech}^{2} \theta_{1}, \\
& \phi_{1}=\left(\frac{k_{1}}{2}\right)^{1 / 2} \operatorname{sech} \theta_{1} .
\end{aligned}
$$

Substituting (C21) and (C22) into (C19) and (C20), we obtain

$$
\begin{aligned}
& \frac{d k_{1}}{d t}=-\frac{\epsilon}{4 k_{1}} \int_{-\infty}^{\infty} R\left[u_{0}\right] \operatorname{sech}^{2} \theta_{1} d \theta_{1}, \\
& \frac{d \xi_{1}}{d t}=4 k_{1}^{2}-\frac{\epsilon}{4 k_{1}^{3}} \int_{-\infty}^{\infty} R\left[u_{0}\right]\left(\theta_{1} \operatorname{sech}^{2} \theta_{1}+\tanh \theta_{1}+\tanh ^{2} \theta_{1}\right) d \theta_{1} .
\end{aligned}
$$

The above results coincide with those derived by IST [14].

[1] G. B. Whitham, Linear and Nonlinear Waves (Wiley, New York, 1974).

[2] Nonlinear Water Waves, edited by $\mathbf{K}$. Horikawa and $\mathrm{H}$. Maruo (Springer-Verlag, New York, 1988).

[3] L. Debnath, Nonlinear Water Waves (Academic, New York, 1994).

[4] J. G. B. Byatt-Smith, J. Fluid Mech. 49, 625 (1971); 182, 485 (1987); 197, 503 (1988); 205, 573 (1989).

[5] C. H. Su and R. M. Mirie, J. Fluid Mech. 98, 509 (1980).

[6] R. M. Mirie and C. H. Su, J. Fluid Mech. 147, 213 (1984).

[7] R. M. Mirie and C. H. Su, J. Fluid Mech. 115, 475 (1982).

[8] J. D. Fenton and M. M. Rienecker, J. Fluid Mech. 118, 411 (1982).

[9] M. Funakoshi and M. Oikawa, J. Phys. Soc. Jpn. 51, 1018 (1982).

[10] Q. Zou and C. H. Su, Phys. Fluids 29, 2113 (1986).

[11] Y. Matsuno, Phys. Rev. E 49, 2091 (1994).

[12] D. J. Kaup, SIAM (Soc. Ind. Appl. Math.) J. Appl. Math. 31, 121 (1976).

[13] D. J. Kaup and A. C. Newell, Proc. R. Soc. London Ser. A 361, 413 (1978).

[14] V. I. Karpman and E. M. Maslov, Phys. Lett. A 60, 307 (1977); Zh. Eksp. Teor. Fiz. 73, 537 (1977) [Sov. Phys. JETP 46, 281 (1977)]; 75, 504 (1978) [48, 252 (1978)].

[15] V. I. Karpman, Phys. Scr. 20, 462 (1979).

[16] E. M. Maslov, Theor. Math. Phys. 42, 237 (1980).

[17] M. Tanaka, J. Phys. Soc. Jpn. 49, 807 (1980).

[18] K. A. Gorshkov and L. A. Ostrovsky, Physica D 3, 428 (1981).

[19] Y. Kodama and M. J. Ablowitz, Stud. Appl. Math. 64, 225 (1981).

[20] R. Grimshaw and H. Mitsudera, Stud. Appl. Math. 90, 75
(1993).

[21] J. P. Keener and D. W. McLaughlin, Phys. Rev. A 16, 777 (1977).

[22] R. L. Herman, J. Phys. A 23, 2327 (1990).

[23] L. A. Kalyakin, Theor. Math. Phys. 92, 736 (1992).

[24] V. V. Konotop and V. E. Vekslerchik, Phys. Rev. E 49, 2397 (1994).

[25] A. Bonderson, M. Lisak, and D. Anderson, Phys. Scr. 20, 479 (1979); J.-C. Fernandez, C. Froeschle, and G. Reinisch, ibid. 20, 545 (1979).

[26] M. Oikawa and N. Yajima, J. Phys. Soc. Jpn. 34, 1093 (1973); Prog. Theor. Phys. Suppl. 55, 36 (1974).

[27] A. C. Newell, in Solitons, edited by R. K. Bullough and P. J. Caudrey (Springer, Berlin, 1980), p. 177.

[28] Yu. S. Kivshar and B. A. Malomed, Rev. Mod. Phys. 61, 763 (1989).

[29] T. B. Benjamin, J. Fluid Mech. 29, 559 (1967).

[30] H. Ono, J. Phys. Soc. Jpn. 39, 1082 (1975).

[31] Y. Matsuno, Bilinear Transformation Method (Academic, New York, 1984).

[32] P. M. Edwin and B. Roberts, Wave Motion 8, 151 (1986).

[33] D. R. Christie, J. Atmos. Sci. 46, 1462 (1989).

[34] A. H. Nayfeh, Perturbation Methods (Wiley, New York, 1973).

[35] A. Jefferey and T. Kawahara, Asymptotic Methods in Nonlinear Wave Theory (Pitman, London, 1982).

[36] Y. Matsuno, J. Phys. A 12, 619 (1979).

[37] Y. Matsuno, J. Phys. A 13, 1519 (1980).

[38] J. P. Keener and D. W. McLaughlin, J. Math. Phys. 18, 2008 (1977).

[39] C. J. Knickerbocker and A. C. Newell, J. Fluid Mech. 98, 803 (1980). 
[40] This result has been obtained by a different method: Y. Matsuno, Phys. Rev. Lett. 73, 1316 (1994).

[41] H. Airault, H. P. McKean, and J. Moser, Commun. Pure Appl. Math. 30, 95 (1977); S. V. Manakov, V. E. Zakharov, L. A. Bordag, A. R. Its, and V. B. Matveev, Phys. Lett. A 63, 205 (1977); M. J. Ablowitz and J. Satsuma, J. Math. Phys. 19, 2180 (1978); J. Satsuma and M. J. Ablowitz, ibid. 20, 1496 (1979); Y. Matsuno, J. Phys. Soc. Jpn. 57, 1577 (1988); 58, 1948 (1989); J. Math. Phys. 31, 2904 (1990); C. P. Gilson and J. J. C. Nimmo, Phys. Lett. A 147, 472 (1990).

[42] R. L. Sachs, SIAM (Soc. Ind. Appl. Math.) J. Math. Anal.
14, 674 (1983).

[43] V. A. Arkad'ef, A. K. Pogrebkov, and M. K. Polivanov, Theor. Math. Phys. 72, 909 (1987).

[44] C. G. Koop and G. Butler, J. Fluid Mech. 112, 225 (1981).

[45] R. I. Joseph, J. Phys. A 10, L225 (1977).

[46] T. Kubota, D. R. S. Ko, and L. D. Dobbs, AIAA J. Hydronautics 12, 157 (1978).

[47] Y. Matsuno, Phys. Lett. A 74, 233 (1979).

[48] Y. Matsuno, J. Phys. Soc. Jpn. 62, 1902 (1993).

[49] F. Calogero and A. Degasperis, Spectral Transform and Solitons I (North-Holland, Amsterdam, 1982). 